\title{
The U.S. Needs a National Human Health Observing System
}

\author{
Paul A. Sandifer ${ }^{1 *}$, Burton H. Singer ${ }^{2}$ and Rita R. Colwell ${ }^{3}$ \\ ${ }^{1}$ Center for Coastal Environmental and Human Health, School of Sciences and Mathematics, College of Charleston, \\ Charleston, SC, United States, ${ }^{2}$ Emerging Pathogens Institute, University of Florida, Gainesville, FL, United States, ${ }^{3}$ Johns \\ Hopkins University Bloomberg School of Public Health, University of Maryland Institute for Advanced Computer Studies, \\ University of Maryland College Park, Baltimore, MD, United States
}

\section{OPEN ACCESS}

Edited by:

Alexandra P. Leader,

Eastern Virginia Medical School,

United States

Reviewed by:

Suffian Hadi Ayub,

MARA University of

Technology, Malaysia

Frederick Ato Armah,

University of Cape Coast, Ghana Teresa Yamana,

Mailman School of Public Health,

United States

*Correspondence:

Paul A. Sandife

sandiferpa@cofc.edu

Specialty section:

This article was submitted to

Public Health Policy,

a section of the journal

Frontiers in Public Health

Received: 05 May 2021

Accepted: 11 August 2021

Published: 06 September 2021

Citation:

Sandifer PA, Singer BH and Colwell RR (2021) The U.S. Needs a National Human Health Observing

System.

Front. Public Health 9:705597. doi: 10.3389/fpubh.2021.705597
The COVID-19 pandemic and increasing frequency and severity of environmental disasters reveal an urgent need for a robust health observing/surveillance system. With the possible exception of Brazil, we know of no such comprehensive health observing capacity. The US should create a national system of linked regionally-based health monitoring systems similar to those for weather, ocean conditions, and climate. Like those for weather, the health observing system should operate continuously, collecting mental, physical, and community health data before, during, and after events. The system should include existing cross-sectional health data surveys, along with significant new investment in regional longitudinal cohort studies. The recently described framework for a Gulf of Mexico Community Health Observing System is suggested as a potential model for development of a nation-wide system.

Keywords: health observing system, health policy, COVID-19, disasters, cohort studies, health surveillance, Gulf of Mexico

\section{INTRODUCTION}

Our objectives in this short communication are to illustrate the overarching need for a national human health observing system, provide an example of what such a system might encompass, and outline two steps, one near-term and one medium- to long-term, that could be taken toward its development.

More than any previous disaster of the twenty or twenty first centuries, the COVID-19 pandemic, and the increasing likelihood of more emerging diseases in the future, have highlighted an urgent, overarching need for comprehensive health surveillance at local to national scales (1-4). The conditions in the US earlier in the pandemic and the current crises in Brazil (5) and India (6) are stark examples of the need. This pandemic, like numerous previous ones, is likely the result of a zoonotic disease, a cross-over from unidentified animals (most probably bats) to humans $(7,8)$. It is far from the last pandemic likely to occur, based on the history of poorly regulated legal and unrestrained illegal wildlife trade that regularly results in human exposure to potentially deadly wildlife diseases (9-12). That, in addition to the exponential global growth in human travel and material transport $(13,14)$, makes the probability very high. The current pandemic is causing high mortality levels and serious physical and mental health impacts in the populations of countries around the world. The adverse health effects are more severe for vulnerable groups, namely the aged, those with underlying health conditions, the disabled, people of minority ethnicity (especially native Americans, African Americans, and Hispanics), people living or working in communal residences such as nursing homes, the military, and prisons, and others. In addition, there is a very serious need to track and alleviate the stresses associated with illness anxiety, management of 
stay-at-home work and education, social distancing, business closures and job losses (15). These factors become more complicated in unrelated but simultaneously occurring lifeand livelihood threatening circumstances, in particular the mandatory evacuations and destruction associated with disasters, notably hurricanes, tropical storms, tornados, floods, and wildfires that occurred at unprecedented levels in 2020 and portend to occur more frequently and severely in the future $(16,17)$. Dealing with potential health impacts, and tracking their effects and treatment over time, of even one such disaster is fraught with difficulty. The possibility of having to deal with multiple and perhaps simultaneously occurring disasters amidst an ongoing pandemic is even more daunting. Of high concern is the likely occurrence of excess morbidity and mortality, i.e., not directly tied to the pandemic but resulting from delayed and/or postponed treatment and preventive medical care for other conditions unrelated to the disaster as occurred following Hurricanes Katrina and Maria (18-20).

\section{POLICY OPTIONS AND IMPLICATIONS}

One recommendation suggested by Gottleib et al. (2) to the CDC (Centers for Disease Control and Prevention) was to "convene an intergovernmental task force, with outside experts as needed and input from states and the health care community, to develop and support a new national surveillance system and data infrastructure for tracking and analyzing COVID-19." Similarly, among recommendations for improving pandemic preparedness and resilience in the U.S., Daszak et al. (21) emphasized strengthening the nation's public health systems and establishment of an independent commission led by the National Academies of Science, Engineering, and Medicine (NASEM) to review the US pandemic response and develop strategies for the future. We agree with these suggestions, but believe they may be overly focused on the current health emergency, namely COVID-19, and insufficiently broad to encompass not only future disease epidemics, but also the aggregated health effects from multiple types of disasters and associated collateral social and economic damage. Acknowledging obstacles that derive from the balkanized US health care system, we recommend establishing regionally-based, interlinked longitudinal cohort studies that will provide early warning and tracking of pandemic disease and other health issues at a country-wide scale. Specifically, we highlight the need for a health observing system that combines both cross-sectional and individual patient data with information from specifically designed longitudinal cohort studies. Unfortunately, no comprehensive health surveillance system that is representative of the population, inclusive of the vulnerable, longitudinal and continuous, and incorporates information from ongoing cross-sectional surveys and other studies, presently exists in the U.S. Regrettably, the national health system in Brazil is being degraded, but it provides examples of what could be done (22). In the U.S., the need for prospective cohort studies for COVID-19 was identified relatively early in the pandemic (4), and repurposing some ongoing longitudinal cohort studies is in process (23). In addition, a few relatively short-term prospective and retrospective cohort studies targeted to COVID-19 in specific localities are underway $(24,25)$, and the National Institutes of Health (NIH) is supporting a National COVID Cohort Collaborative. The latter is to serve as "a secure portal for patient-level COVID-19 clinical data" (26), and it may be possible to utilize the All of Us national cohort study (https://www.nih.gov/news-events/news-releases/allus-research-program-launches-covid-19-research-initiatives). Unfortunately, the sampling being done by the All of Us study lacks a statistically-based approach, thus limiting the epidemiological utility (27). Thus, a comprehensive, prospective cohort-based system at the regional to the national scale is not now available. Lack of a health surveillance capacity hampers all efforts, from monitoring numbers of infections, to effects of the disease and treatment, long-term and unforeseen consequences, and even the ability to determine when/if a recovery has begun and at what rate of progress. Of notable importance is the need to monitor vaccine demand, distribution and uptake in the event it becomes necessary to administer annual vaccinations basis against additional variants of SARS-CoV-2 virus, the causative agent of COVID-19.

Prior to onset of the COVID-19 pandemic, with its massive health, economic, and social impact, a diverse team of scientists and health professionals with expertise in disaster response, public health, medicine, epidemiology, observing systems, and other relevant areas of environmental health science had focused on health information needed for the disaster-prone Gulf of Mexico region. In addition to a series of environmental disasters, including Hurricane Katrina, the Deepwater Horizon oil spill, and six land-falling tropical cyclones in 2020 alone, Gulf residents continue to suffer long-term health, socio-economic, and educational disparities (28-30). Climate change, subsidence, development pressures, harmful algal blooms and other oceanassociated health threats, and the COVID-19 pandemic are examples of recent calamities that are expected to increase in occurrence and severity. Recent research revealed a critical lack of availability of baseline health data captured before, during, and continuing long after a disasters. It was concluded that ongoing health monitoring is essential to reduce and manage health impacts of future disasters. In this regard, (31) highlighted the crucial importance of acquiring and using baseline scientific information in any response to a societal crisis. In summary, to address the need outlined in this communication the Research Board of the Gulf of Mexico Research Initiative commissioned a study to develop a framework for a health observing system. An interdisciplinary team undertook this assignment under the leadership of P. Sandifer and B. Singer. The work was accomplished through two expert workshops, consultations with knowledgeable individuals, review of an extensive body of literature and ongoing health surveys, and iterative design and writing efforts, and was informed by existing environmental observing systems $(32,33)$.

The resulting design builds on existing national health surveys and studies $(32,33)$. Most importantly, it has added several longitudinal cohort studies focused explicitly on vulnerable coastal areas and includes specific disaster-responsive 


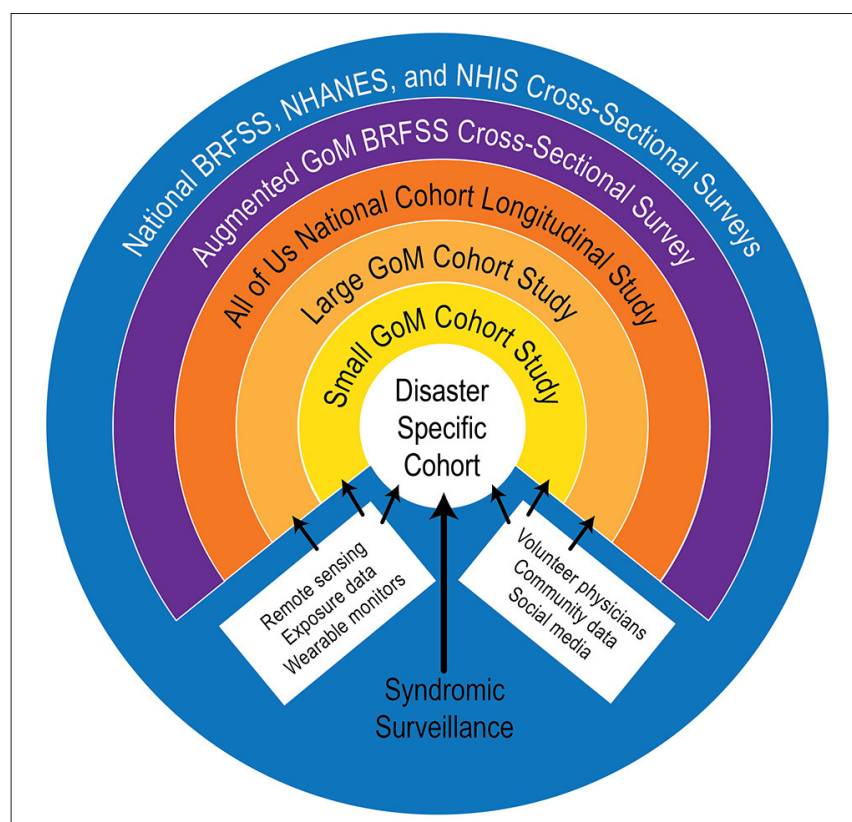

FIGURE 1 | Schematic framework for a Gulf of Mexico Community Health Observing System [from Sandifer et al. (33)].

components, representative and targeted population sampling, and self-reported and clinically-derived demographic, psychosocial, and physical health data. The health observing system framework, illustrated conceptually in Figure 1, takes advantage of available and developing technologies such as electronic health records, wearable health devices, remote sensing, advances in genomic and other omic sciences and embraces community-based participatory research approach as an integral operating principle. Importantly, the proposed Gulf health observing system incorporates measures of chronic and cumulative stress that utilize biomarkers to identify allostatic load, an index of toxic stress. Toxic stress is known to be a pervasive problem associated with disasters and other traumatic events and it often leads to long-term psychosocial and physiological pathologies (33-39). In fact, harmful effects of massive stress caused by childhood exposure to the Holocaust was recorded by Holocaust survivors more than 70 years after the fact (39). Peters et al. (40) posited that pre-disease psychosocial assessments of chronic stress could help guide COVID-19 treatments.

The Gulf of Mexico Health Observing System (GoM CHOS) framework design is based, in part, on the atmospheric and oceanic observing systems that underlie modern weather, climate, and marine forecasting capabilities, upon which hundreds of millions to billions of people depend daily. These environmental observing systems enable society to mitigate effects of extreme weather events and resulting costly health and socio-economic impacts at local to global scales. Using the GoM CHOS as a model for pilot studies and building out to a regional framework, as employed by the US Integrated Ocean Observing System (IOOS) (https://ioos.noaa.gov/regions/), could provide a nation-wide health observing system of unparalleled capacity.
As noted in Sandifer et al. $(32,33)$, risks to participants in a health observing system are expected to be relatively minor and include lapses in data security, anxiety or discomfort in providing personal information or associated with clinical visits and/or biospecimen collection, and programmatic issues such as continuity, funding, and management. Significant participant benefits include regular medical checkups, continued health monitoring over time, increased personal and community resilience, and enhanced personal preparedness to deal with health consequences of future disasters and other traumatic events. At a larger scale, information from health observing systems can lead to development of public health strategies that help prevent or mitigate disaster-associated health hazards and propel innovation in medical research and practice. Other larger scale advantages deriving from a nation-wide health observing system are presented in the conclusions section of this communication.

Establishment of a nation-wide structure of linked regional health observing systems patterned after the GoM CHOS framework or some other models that include both crosssectional and representative longitudinal cohort components can be a major step toward a national health observing/surveillance capability. Connecting regional health observing units to academic health centers, the private sector, and data management centers will increase the reach and utility of the health observing system significantly. Clinical data collection components can include testing for SARS-CoV-2 or other potential diseases of known concern, and new sampling and diagnostic technologies for emerging diseases and health issues can be incorporated in existing longitudinal cohorts. Ongoing longitudinal cohorts will provide mechanisms for tracking prevalence and health effects over time, as well as association or interaction with other health factors including psychosocial and physiological stress, effectiveness of vaccines, and other preventative methods, and treatments. A national health observing system can provide data needed by public health officials, clinicians, researchers, and the public to monitor progress in managing health effects of future pandemics, environmental disasters, and economic disruptions.

\section{ACTIONABLE RECOMMENDATIONS}

We recommend the following short- and medium-to longterm actions:

1) Short-term: As a first step, the Federal Government or Congress should authorize a Federal agency (or group of agencies) to undertake a health observing system pilot program and it could be in the Gulf of Mexico region to take advantage of the Gulf of Mexico Community Health Observing System framework. Wherever and however conducted, the purpose of the pilot would be "to learn by doing," to establish a strong base of information for designing and building a national network of regional health observatories. Although cost estimates were not a part of the original study, a rough "guestimate" of funding required for a Gulf region health observing system is ca. $\$ 10 \mathrm{M} /$ year. Better estimates will depend on implementation decisions (33). Almost certainly, a robust pilot project could be undertaken 
with financial support at this level, although a system at national scale would require considerably more funding. In this light, we note that in 2016, before the pandemic, the U.S. Congress authorized funding of $\$ 1.5 \mathrm{~B}$ over 10 years for the NIH All of Us study (https://allofus.nih.gov/news-events/ press-kit/all-us-research-program-backgrounder).

2) Medium to long-term: The Federal Government, with leadership of the CDC, NIH, and NSF and participation of the NASEM, should establish a Commission or Committee to make recommendations to the President and the Congress for creation and long-term funding of a national system of regional health observing systems focused on pandemics and disasters. The Commission or Committee should comprise public health and biomedical experts with relevant expertise from Federal Government health agencies, State Health Departments, public health and medical experts from academic institutions, public and private health care providers, computational data specialists, and others as may be required.

\section{CONCLUSIONS}

The COVID-19 pandemic and the increasing frequency and severity of environmental disasters make obvious the urgent need for a robustly operational health observing system for the U.S. A comprehensive health observing capacity does not exist and should be developed. A regionally based, nationally linked system, as outlined in this communication, can serve to gather the health information, including mental, physical and community health data, in a representative sampling of the nation's population, including the susceptible or vulnerable, with respect to effects of pandemics and other disasters. The database would be available to public health officials, medical practitioners, disaster preparedness planners, biomedical researchers, and others to improve health preparedness and response. Multiple health observing systems would create comparable, ongoing health data streams that could improve targeting of investments for disaster preparedness, response, and prevention, there by

\section{REFERENCES}

1. Fauci AS, Lane HC, Redfield RR. Covid-19 - navigating the uncharted. N Engl J Med. (2020) 382:1268-9. doi: 10.1056/NEJMe2002387

2. Gottlieb S, Rivers C, McClellan MB, Silvis L, Watson C. National Coronavirus Response: A Road Map to Reopening. Washington, DC: American Enterprise Institute (2020).

3. Kissler SM, Tedijanto C, Goldstein E, Grad YH, Lipsitch M. Projecting the transmission dynamics of SARS-CoV-2 through the postpandemic period. Science. (2020) 368:860-8. doi: 10.1126/science.ab b5793

4. Lipsitch M, Swerdlow DL, Finelli L. Defining the epidemiology of Covid-19 - studies needed. N Engl J Med. (2020) 382:11946. doi: 10.1056/NEJMp2002125

5. Castro MC, Kim S, Barberia L, Ribiero AF, Gurzenda S, Ribiero KB, et al. Spatiotemporal pattern of COVID-19 spread in Brazil. Science. (2021) 372:821-6. doi: 10.1126/science.abh1558

6. Mallapaty S. India's massive covid surge puzzles scientists. Nature. (2021) 592:667-8. doi: 10.1038/d41586-021-01059-y reducing future expenditures for long-term health care related to disasters. Also, such systems could provide earlier and stronger warnings of impending epidemics, as well as tracking of possible long-term effects and contacts, allowing more targeted and possibly less severe responses, which would reduce economic and other societal impacts. Although aspirational at present, a national health observing system is feasible. Considering the high annual costs of disasters and the massive cost of the COVID-19 pandemic, not taking action to improve health monitoring will be far more expensive.

\section{AUTHOR CONTRIBUTIONS}

PS, BS, and RC were responsible for concept formulation. PS had primary funding and writing responsibilities. All authors contributed ideas, reviewed, and approved the manuscript.

\section{FUNDING}

The work was supported in small part by contract \# C231826 between the Gulf of Mexico Alliance, on behalf of the Gulf of Mexico Research Initiative, and the College of Charleston and by the National Institute of Environmental Health Sciences of the National Institutes of Health under Award Number P01ES028942 to the University of South Carolina and through a sub award to the College of Charleston, and National Science Foundation Grant No. CCF1918749 to the University of Maryland College Park. No sponsor played any role in the study design, collection, analysis, and interpretation of data, writing of the manuscript, or in the decision to submit the paper for publication.

\section{ACKNOWLEDGMENTS}

We thank Drs. John Farrington, David Halpern, Charles Wilson for helpful discussion during development of this paper.

7. Beyer RM, Mancia A, Mora C. Shifts in global bat diversity suggest a possible role of climate change in the emergence of SARS-CoV-1 and SARS-CoV-2. Sci Tot Environ. (2020) 767:134413. doi: 10.1016/j.scitotenv.2021.145413

8. Swelum AA, Shafi ME, Albaqami NM, El-Saadony MT, Elsify A, Abdo M, et al. COVID-19 in human, animal, and environment: a review. Front Vet Sci. (2020) 7:578. doi: 10.3389/fvets.2020.00578

9. Gomez A, Aguirre AA. Infectious diseases and the illegal wildlife trade. Anim Biodiv Emerg Dis. (2008) 1149:16-9. doi: 10.1196/annals.1428.046

10. Smith KM, Zambrana-Torrelio C, White A, Asmussen M, Machalaba C, Kennedy S, et al. Summarizing US wildlife trade with an eye toward assessing the risk of infectious disease introduction. Ecohealth. (2017) 14:2939. doi: 10.1007/s10393-017-1211-7

11. Can OE, D'Cruze N, MacDonald DW. Dealing in deadly pathogens: taking stock of the legal trade in wildlife and potential risks to human health. Glob Ecol Conserv. (2019) 17:e00515. doi: 10.1016/j.gecco.2018. e00515

12. Dobson AP, Pimm S, Hannah L, Kaufman L, Ahumada JA, Ando AW, et al. Ecology and economics for pandemic prevention. Science. (2020) 369:379381. doi: 10.1126/science.abc3189 
13. Morand S, Walther BA. The accelerated infectious disease risk in the Anthropocene: more outbreaks and wider global spread. bioRxiv [Preprint]. (2020). doi: 10.1101/2020.04.20.049866

14. Ugur NG, Akviyik A. Impact of COVID-19 on global tourism industry: a cross-regional comparison. Tour Manage Perspect. (2020) 36:100744. doi: 10.1016/j.tmp.2020.100744

15. Bavli I, Sutton B, Galea S. Harms of public health interventions against covid19 must not be ignored. BMJ. (2020) 371:m40704. doi: 10.1136/bmj.m4074

16. Pei S, Dahl KA, Yamana TK, Licker B, Shaman J. Compound risks of hurricane evacuation amid the COVID-19 pandemic in the United States. GeoHealth. (2020) 4:e2020GH000319. doi: 10.1029/2020GH000319

17. NOAA (National Oceanic and Atmospheric Administration). Billion Dollar Weather and Climate Disasters: Overview. (2021). Available online at: https:// www.ncdc.noaa.gov/billions/ (accessed July 30, 2021).

18. Stephens KU, Grew D, Chin K, Kadetz P, Greenough G, Burkle FMJr, et al. Excess mortality in the aftermath of Hurricane Katrina: a preliminary report. Disaster Med Public Health Preparedness. (2007) 1:15-20. doi: 10.1097/DMP.0b013e3180691856

19. GWU (George Washington University). Ascertainment of the Estimated Excess Mortality from Hurricane Maria in Puerto Rico. Washington, DC (2018). Available online at: https://publichealth.gwu.edu/sites/default/files/ downloads/projects/PRstudy/Acertainment\%20of\%20the\%20Estimated $\% 20$ Excess $\% 20$ Mortality\%20from $\% 20$ Hurricane $\% 20$ Maria $\% 20$ in $\% 20$ Puerto \%20Rico.pdf/ (accessed June 9, 2020).

20. Kishore N, Marques D, Mahmud A, Kiang MV, Rodriguez I, Fuller A, et al. Mortality in Puerto Rico after Hurricane Maria. N Engl J Med. (2018) 379:162-70. doi: 10.1056/NEJMsa1803972

21. Daszak P, Keusch GT, Phelan AL, Johnson CK, Osterholm MT. Infectious disease threats: a rebound to resilience. Health Aff. (2021) 40:20411. doi: 10.1377/hlthaff.2020.01544

22. Castro MC, Massuda A, Almeida G, Menezes-Filho NA, Andrade MV, de Souza Noronha KVM, et al. Brazil's unified health system: the first 30 years and prospects for the future. Lancet. (2019) 394:345-56. doi: 10.1016/S0140-6736(19)31243-7

23. Abbott A. Thousands of people will help scientists to track the long-term health effects of the coronavirus crisis. Nature. (2020). Available online at: nature.com/articles/d41586-020-01643-8 (accessed June 9, 2020).

24. Fagherazzi G, Fischler A, Betsoni F, Vaillant M, Ernes I, Masi S, et al. Protocol for a prospective cohort of people with COVID-19 and their household members to study factors associated with disease severity. BMJ Open. (2020) 10:e041834. doi: 10.1136/bmjopen-2020-041834

25. Zhou F, Yu T, Du R, Fan G, Liu Y, Liu Z, et al. Clinical course and risk factors for mortality of adult inpatients with COVID-19 in Wuhan, China: a retrospective cohort study. Lancet. (2020) 395:105462. doi: 10.1016/S0140-6736(20)30566-3

26. NASEM (National Academies of Science, Engineering, and Medicine). Critical Findings on COVID-19. Selected publications of the National Academies of Science, Engineering, and Medicine. Washington, DC (2021).

27. All of Us Research Program, I.nvestigators. The "All of Us" research program. N Engl J Med. (2019) 381:668-76. doi: 10.1056/NEJMsr1809937

28. Lichtveld MY, Arosemena FA. Resilience in the aftermath of the Gulf of Mexico oil spill: an academic-community partnership to improve health education, social support, access to care, disaster preparedness. In: International Oil Spill Conference Proceedings. Savannah, GA (2014). p. 156-69.

29. Lichtveld M, Sherchan S, Gam KB, Kwok RK, Mundorf C, et al. The Deepwater Horizon oil spill through the lens of human health and the ecosystem. Curr Environ Health Rep. (2016) 3:370-8. doi: 10.1007/s40572-016-0119-7

30. Slack T, Kroeger RA, Stroop S, Keating KS, Sury J, Brooks J, et al. Deepwater Horizon oil spill exposure and child health: a longitudinal analysis. Popul Environ. (2021) 42:477-500. doi: 10.1007/s11111-02000354-6
31. Colwell RR, Machlis GE. Science during crisis: best practices, research needs, and policy priorities. American Academy of Arts and Sciences, Cambridge, MA. (2019) 32pp.

32. Sandifer P, Knapp L, Lichtveld M, Manley R, Abramson D, et al. Framework for a community health observing system for the Gulf of Mexico region: preparing for future disasters. Front Public Health. (2020) 8:578463. doi: 10.3389/fpubh.2020.578463

33. Sandifer P, Knapp L, Lichtveld M, Manley R, Abramson D, et al. A Conceptual Framework for a Community Health Observing System for the Gulf of Mexico Region. Gulf of Mexico Research Initiative Core Synthesis Area 4. MississippiAlabama Sea Grant Consortium Technical Report MASGR-20-026. National Sea Grant Library No. GOMSG-20-001 (2020).

34. Seeman TE, Singer BH, Rowe JW, Horwitz RI, McEwen BS. Price of adaptation -allostatic load and its health consequences. MacArthur studies of successful aging. Arch Intern Med. (1997) 157:2259-68. doi: 10.1001/archinte.1997.00440400111013

35. McEwen BS. Protective and damaging effects of stress mediators: central role of the brain. Dial Clin Neurosci. (2006) 8:36781. doi: 10.31887/DCNS.2006.8.4/bmcewen

36. Juster, R.-P., Bizik G, Picard M, Arsenault-Lapierre G, Sindi S, et al. A transdisciplinary perspective of chronic stress in relation to psychopathology throughout life span development. Dev Psychopathol. (2011) 23:72576. doi: 10.1017/S0954579411000289

37. Sandifer PA, Knapp LC, Collier TK, Jones AL, Juster R-P, Kelble CR, et al. A conceptual model to assess stress-associated health effects of multiple ecosystem services degraded by disaster events in the Gulf of Mexico and elsewhere. GeoHealth. (2017) 1:17-36. doi: 10.1002/2016GH0 00038

38. Sandifer PA, Walker AH. Enhancing disaster resilience by reducing stress-associated health impacts. Front Public Health. (2018) 6:373. doi: 10.3389/fpubh.2018.00373

39. Fnaskova M, Riha P, Preiss M, Bob P, Necasova M, Koritakova E, et al. Lifelong impact of extreme stress on the human brain: Holocaust survivors. Neurobiol Stress. (2021) 14:100318. doi: 10.1016/j.ynstr.2021.100318

40. Peters EMJ, Schedlowski M, Watzl C, Gimsa U. To stress or not to stress: brain-behavior-immune interaction may weaken or promote the immune response to SARS-CoV-2. Neurobiol Stress. (2021) 14:100296. doi: 10.1016/j.ynstr.2021.100296

Author Disclaimer: The content of this paper is solely the responsibility of the authors and does not necessarily represent the official views of the Gulf of Mexico Alliance, the Gulf of Mexico Research Initiative, the National Institute of Environmental Health Sciences, the College of Charleston the National Science Foundation, or the University of Maryland College Park.

Conflict of Interest: The authors declare that the research was conducted in the absence of any commercial or financial relationships that could be construed as a potential conflict of interest.

Publisher's Note: All claims expressed in this article are solely those of the authors and do not necessarily represent those of their affiliated organizations, or those of the publisher, the editors and the reviewers. Any product that may be evaluated in this article, or claim that may be made by its manufacturer, is not guaranteed or endorsed by the publisher.

Copyright (c) 2021 Sandifer, Singer and Colwell. This is an open-access article distributed under the terms of the Creative Commons Attribution License (CC BY). The use, distribution or reproduction in other forums is permitted, provided the original author(s) and the copyright owner(s) are credited and that the original publication in this journal is cited, in accordance with accepted academic practice. No use, distribution or reproduction is permitted which does not comply with these terms. 